\title{
The Effect of Periarcuate Lesions in the Monkey on the Performance of Symmetrically and Asymmetrically Reinforced Visual and Auditory Go, No-Go Tasks
}

\author{
Michael Petrides \\ Department of Psychology, McGill University, Montreal, Quebec H3A 1B1, and the Montreal Neurological \\ Institute, McGill University, Montreal, Quebec H3A 2B4, Canada
}

\begin{abstract}
Monkeys with lesions restricted to the periarcuate region of the frontal cortex were impaired on go, no-go tasks in which, depending on the stimulus present on any given trial, they were rewarded either for pushing a manipulandum or for withholding this response for a given period of time. By contrast, these animals were able to learn at a normal rate go, no-go tasks in which they were rewarded if they responded in the presence of a "positive" stimulus, but not in the presence of a "negative" stimulus. In the latter situation, responding in the presence of the "negative" stimulus was extinguished through non-reward, whereas the tasks on which impairments were demonstrated required solution on the basis of a conditional rule: If stimulus $A$, respond, and if stimulus $B$, withhold responding, to receive reward. Because the 2 experimental situations were identical in every respect except for the one critical difference in the testing procedure, the present set of experiments provides a powerful demonstration of the involvement of the periarcuate cortex in conditional learning with exteroceptive stimuli.
\end{abstract}

Damage to the dorsolateral frontal cortex of the monkey, particularly the periarcuate region (rostral area 6 and area 8), impairs performance on acoustically and visually cued conditional position-response tasks (Goldman and Rosvold, 1970; Lawicka et al., 1975; Milner et al., 1978; Podbros et al., 1980; Stamm, 1973). The spatial nature of either the cues or the responses in these tasks has often been emphasized in the various attempts that have been made to interpret these deficits (e.g., Konorski, 1975; Milner et al., 1978; Stamm, 1973). Such attempts, however, cannot account for the fact that monkeys with periarcuate lesions are only slightly impaired (Goldman and Rosvold, 1970; Goldman et al., 1971) or are unimpaired (Goldman et al., 1971; Rosenkilde et al., 1981) on various tasks involving clearly spatial responses. Similarly, such explanations cannot account for the deficit of monkeys with such lesions on a completely nonspatial compound task in which monkeys have to respond when an auditory and a visual cue are presented together, but to refrain from responding when either of these stimuli is presented by itself (Petrides and Iversen, 1978; Van Hoesen et al., 1980).

A broader conception of the role of the periarcuate cortex can be suggested if the above findings are considered in association with the following facts: First, it has been shown that patients with frontal-lobe lesions are severely impaired in mastering conditional associative-learning tasks in which they have to learn

\footnotetext{
Received Oct. 8, 1985; revised Dec. 19, 1985; accepted Dec. 26, 1985.

The Natural Sciences and Engineering Research Council of Canada supported this research through Grant A7466 to the author. I thank K. Morris for testing the animals and V. Baillargeon for drawing Figures 3 and 8.

Correspondence should be addressed to the author at Department of Psychology, McGill University, 1205 Dr. Penfield Avenue, Montreal, Quebec, H3A 1B1, Canada.

Copyright (C) 1986 Society for Neuroscience $0270-6474 / 86 / 072054-10 \$ 02.00 / 0$
}

arbitrary associations between a set of stimuli and a set of responses (Petrides, in Milner, 1982; Petrides, 1985a). Second, it has been known for some time that input from visual, auditory, and somaesthetic "association" cortex converges in and around the arcuate sulcus (Barbas and Mesulam, 1981; Chavis and Pandya, 1976; Pandya and Kuypers, 1969; Petrides and Pandya, 1984; Van Hoesen et al., 1972) and that the periarcuate cortex is connected to the motor cortex as well as subcortical regions involved in response control (Astruc, 1971; Godschalk et al., 1984; Kuypers and Lawrence, 1967; Matsumura and Kubota, 1979; Muakkassa and Strick, 1979; Pandya and Vignolo, 1971). On the basis of these observations, the periarcuate cortex may be thought of as playing a critical role in the control of conditional associative tasks in which a set of exteroceptive stimuli have to be linked to a set of arbitrary responses (Petrides, 1982). The deficits on the acoustically and visually cued position-response tasks can be viewed as special instances of a more general impairment following periarcuate lesions rather than as deficits attributable to the spatial nature of the stimuli or the responses.

In a conditional learning situation, the subject is faced with a set of stimuli and a set of arbitrary responses that bear no relation to the stimuli, and he has to learn the conditional rule: If stimulus A is presented, select response $X$, and if stimulus B is presented, select response $Y$. Conditional learning is thus fundamentally different from the kind of learning that is required, for instance, in a standard discrimination task. In such a task, the monkey is rewarded for making a certain response (e.g., displacing the stimulus) to the "positive," but not the "negative," stimulus. In this way, producing a given response to the "positive" stimulus is strengthened through reinforcement, whereas producing the same response to the "negative" stimulus is gradually extinguished through non-reward. This is in sharp contrast to the conditional learning situation in which all stimuli are "positive," i.e., they all lead to reward if the appropriate response is produced.

The experiments reported in this communication were designed to test the predictions of the hypothesis that the periarcuate cortex is involved in conditional learning on 2 distinct types of a go, no-go task in which animals are trained to make a response in the presence of one stimulus, but not in the presence of another stimulus. It has been shown in a number of investigations (see Dabrowska, 1971, 1972; Konorski, 1972, 1975 ) that, in these situations, a go, no-go task in which the nogo response is not rewarded (asymmetrical reinforcement) is very different from a go, no-go task in which the no-go response is rewarded (symmetrical reinforcement). The absence of response to the negative stimulus, in the first case, is simply the result of lack of reinforcement. Expressed differently, it is the result of learning to ignore (extinguish responding to) a nonrewarded stimulus. In the second type of go, no-go task in which the animal is rewarded both for responding to the go stimulus 


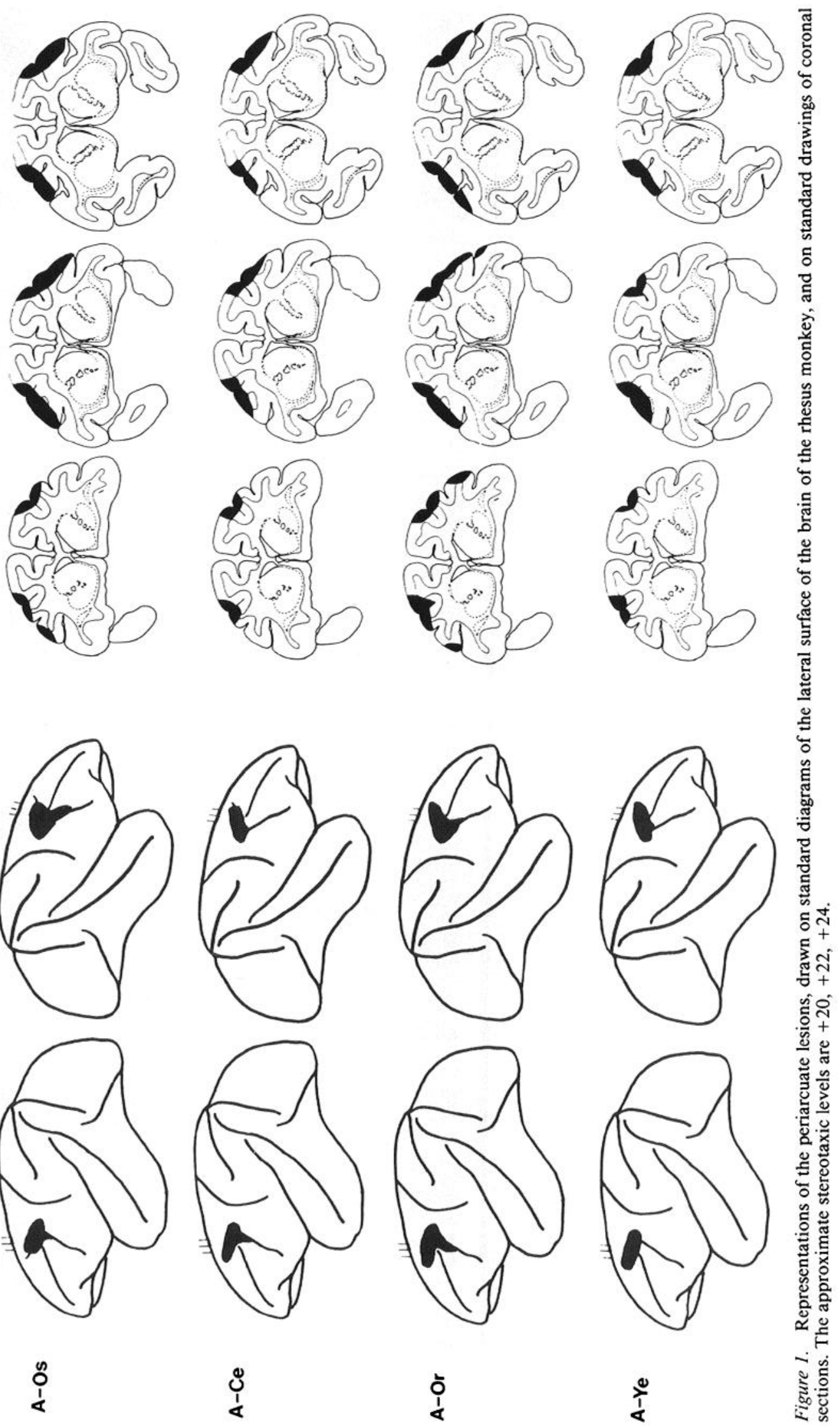


P-TO
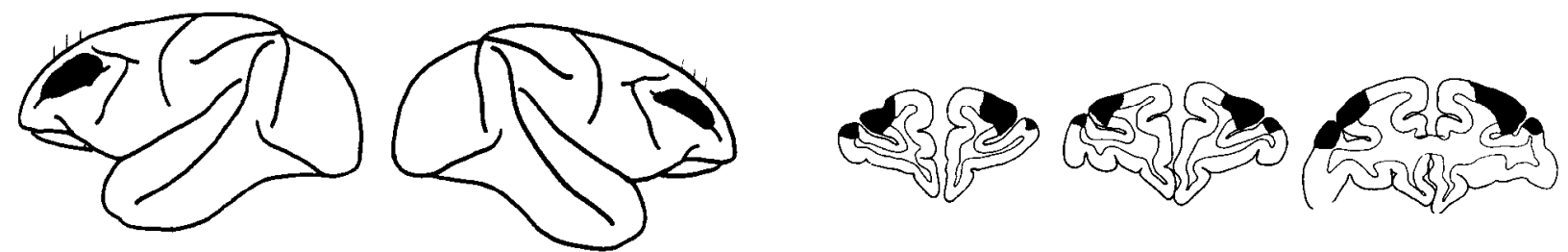

P-Ne
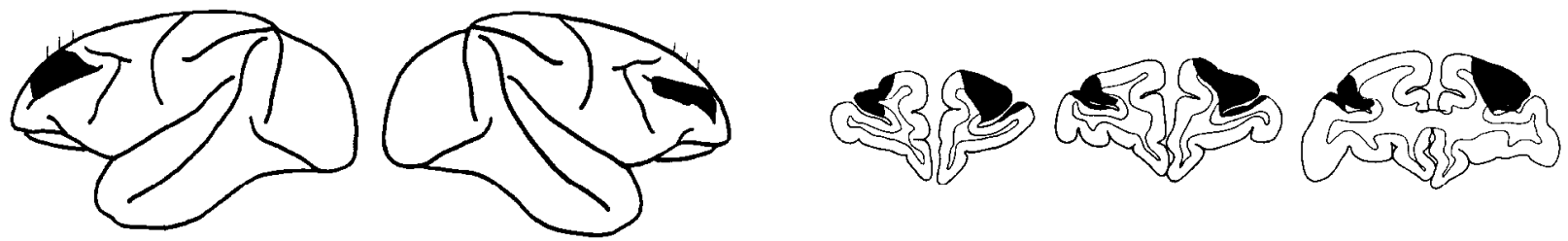

P-Wo
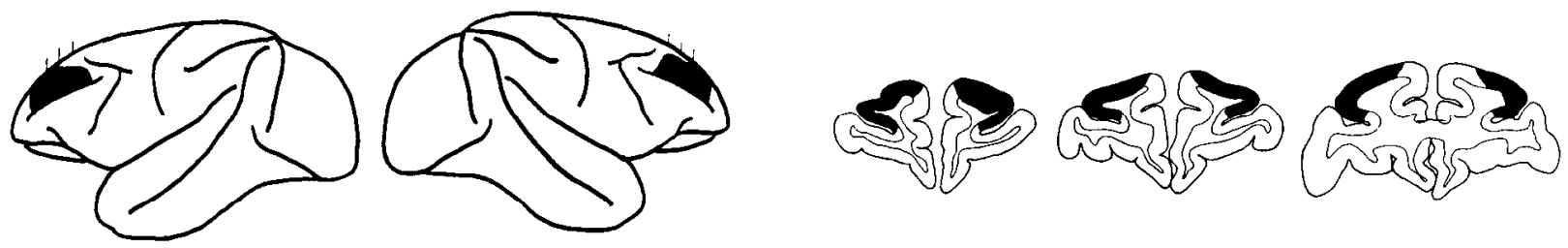

Figure 2. Representations of the periprincipalis lesions drawn on standard diagrams of the lateral surface of the brain of the rhesus monkey, and on standard drawings of coronal sections. The approximate stereotaxic levels are $+30,+33,+36$.

and for withholding its responses to the no-go stimulus for a certain period of time, the no-go response cannot simply be the result of extinction of responding through non-reward. Instead, as in conditional learning, the subject must acquire, and perform according to, the conditional rule: If stimulus $A$ is presented, go, and if stimulus B is presented, withhold responding to receive the reward. The hypothesis proposed above predicts that monkeys with periarcuate lesions will be impaired on a go, nogo task that is symmetrically reinforced, but will not be impaired on an asymmetrically reinforced one.

\section{Materials and Methods}

\section{Subjects and surgical procedure}

The subjects were 10 male rhesus monkeys (Macaca mulatta) weighing from 3.6 to $5 \mathrm{~kg}$ at the time of surgery. Three of these animals served as unoperated controls. Four monkeys were given a one-stage bilateral ablation of the periarcuate region of the frontal cortex. These lesions were intended to be similar to those investigated by Petrides and Iversen (1978), and were to include the cortex immediately surrounding, and lying within, the superior ramus, the spur, and the uppermost part of the inferior ramus of the arcuate sulcus. In removing cortex from the rostral bank of the lower ramus of the arcuate sulcus, great care was taken not to damage or undercut the cortex of the inferior convexity, a part of the frontal cortex that has been associated with the perseverative behavior characteristic of large frontal-lobe lesions (Iversen and Mishkin, 1970). Three animals received one-stage bilateral ablations intended to include cortex in the sulcus principalis and the cortex lying dorsal to it. The posteriormost part of the sulcus principalis was to be spared because its connections share certain features with those of the periarcuate region (Barbas and Mesulam, 1981; Pandya and Kuypers, 1969; Pandya et al., 1971). Care was also taken not to damage or undercut the cortex of the inferior convexity in performing these latter lesions. All operations were carried out by standard aseptic techniques for subpial aspiration of the cortex.

\section{Histological procedure}

At the completion of the present and other experiments, the animals were sacrificed with an overdose of Nembutal, and their brains fixed in $10 \%$ Formalin. A macroscopic examination that included drawings of the lesions, as seen from the surface of the brain, was carried out. Frozen sections were cut at $30 \mu \mathrm{m}$, and every tenth section from the area that was removed was kept for staining with thionine and for microscopic examination. Representations of the lesions are shown in Figures 1 and
2. The periarcuate lesions were as intended, except that there was a considerable amount of sparing of the cortex in the banks of the sulcus. The lesions of the cortex in and above the sulcus principalis were also as intended, except that a variable amount of the cortex in this sulcus was also spared. The cortex of the inferior prefrontal convexity was not damaged or undercut in any of the lesions.

\section{Experiment 1: visual discrimination I and reversal I}

In this experiment, the animals were trained preoperatively on a visual object discrimination task and were retested, after operation, for retention of this discrimination as well as for learning its reversal. The purpose of the experiment was to establish that the animals could discriminate between 2 objects that were to be subsequently used as the stimuli in a symmetrically reinforced go, no-go task (Experiment 2). Normal performance by the periarcuate monkeys on the visual discrimination would suggest that any deficits that might be found on the go, no-go task could not be interpreted in terms of visual perceptual impairments. Also, normal performance on the reversal part of the discrimination would provide a bchavioral confirmation that the lesions had not interfered with the function of the inferior convexity of the prefrontal cortex, an area that has been linked to the perseveration seen on discrimination-reversal tasks after frontal-lobe lesions (e.g., Iversen and Mishkin, 1970).

\section{Procedure}

Testing was conducted in the Wisconsin General Testing Apparatus (WGTA). When the opaque screen, which separated the monkey's compartment from the testing area, was raised, the animal was faced with a test-board with 2 foodwells $(25.5 \mathrm{~cm}$ apart). Each foodwell was covered with 1 of 2 objects stuck onto white plaques $(6 \times 6 \mathrm{~cm})$. The objects were a red cross - the positive stimulus that always had a peanut under it-and a yellow, oval-shaped object-the negative stimulus that did not cover a peanut. Thirty trials were administered/d. The position of the objects over the foodwells on any given trial was determined according to a random, but balanced, order (Gellermann schedule), i.e., with the restriction that within each block of 10 trials, the positive and negative objects appeared an equal number of times over each of the 2 foodwells. A trial was initiated when the opaque scrcen was raiscd. When the monkey had chosen one of the stimuli by uncovering one of the 2 foodwells, the trial was terminated, and the screen lowered. If the monkey displaced the positive stimulus when the screen was raised, it found a peanut under it, but not if it displaced the negative stimulus. The non-correction technique was used throughout testing, i.e., the next trial in the sequence was administered irrespective of whether the animal's response had been correct or not. Testing continued until the 


\begin{tabular}{|c|c|c|c|c|}
\hline Subject & $\begin{array}{l}\text { Visual dis- } \\
\text { crimination I } \\
\text { (preoperative } \\
\text { learning) } \\
\text { Experiment } 1\end{array}$ & $\begin{array}{l}\text { Visual dis- } \\
\text { crimination } \\
\text { reversal I } \\
\text { Experiment } 1\end{array}$ & $\begin{array}{l}\text { Visual dis- } \\
\text { crimination II } \\
\text { (postoperative } \\
\text { learning) } \\
\text { Experiment } 3\end{array}$ & $\begin{array}{l}\text { Visual dis- } \\
\text { crimination } \\
\text { reversal II } \\
\text { Experiment } 3\end{array}$ \\
\hline N-He. & 120 & 150 & 60 & 90 \\
\hline N-Bo. & 150 & 60 & 60 & 90 \\
\hline N-Sa. & 120 & 150 & 60 & 60 \\
\hline P-To. & 150 & 180 & 120 & 150 \\
\hline P-Ne. & 90 & 90 & 60 & 90 \\
\hline P-Wo. & 120 & 180 & 60 & 60 \\
\hline A-Os. & 150 & 180 & 60 & 30 \\
\hline A-Ce. & 120 & 90 & 30 & 60 \\
\hline A-Or. & 210 & 270 & 120 & 60 \\
\hline A-Ye. & 90 & 120 & 30 & 30 \\
\hline
\end{tabular}

$\mathrm{N}$, Normal monkeys; $\mathrm{P}$, monkeys with periprincipalis lesions; $\mathrm{A}$, monkeys with periarcuate lesions. Trials to criterion are not included in the numbers given above.

animals reached a learning criterion of $90 \%$ correct responses on each of 2 consecutive days. After reaching criterion, each animal was allowed a 2 week rest, and, at the end of this period, it was again retested to criterion. Surgery for the animals in the operated groups was carried out at this stage. Two weeks after surgery, the monkeys were retested on the visual discrimination task, and on the day following completion of criterion testing, training on the reversal of the discrimination was initiated. The yellow, oval-shaped object was now the positive stimulus and the red cross the negative stimulus. The testing procedure and criterion of learning were otherwise identical to those used in the original discrimination test.

\section{Results}

As can be seen in Table 1, there were no significant differences between groups on the number of trials needed to learn the visual discrimination preoperatively (Kruskal-Wallis test; $H=0.35, d f=2$, n.s.). All animals retained the discrimination, reaching criterion at once, when they were retested 2 weeks after having learned it and 2 weeks after the operation. On learning the reversal of the visual discrimination, there were again no significant differences between the groups (Kruskal-Wallis test; $H=$ $1.05, d f=2$, n.s.).

\section{Comment}

The results of this experiment show that monkeys with periarcuate lesions are not impaired in postoperative retention of a visual object discrimination and that they can reverse this discrimination at a normal rate, exhibiting none of the perseverative problems characteristic of monkeys with lesions to the inferior convexity of the frontal cortex. These data are consistent with the findings of Goldman and Rosvold (1970), who reported no deficits after periarcuate lesions on a visual pattern discrimination, and they demonstrate that the ability to discriminate between visual stimuli is not affected by such lesions. The normal performance of periarcuate monkeys on the reversal of the object discrimination task is also consistent with the results of an earlier investigation (Goldman et al., 1971) in which lesions to the arcuate cortex did not impair the reversal of a place discrimination.

\section{Experiment 2: symmetrically reinforced visual go, no-go task $I$}

The purpose of this experiment was to test the prediction that monkeys with periarcuate lesions would be impaired in learning a symmetrically reinforced go, no-go task. The animals were to be trained to respond when 1 of 2 objects was shown, and to withhold responding for $5 \mathrm{sec}$ in the presence of the other object in order to receive the reward.

\section{Procedure}

Testing was conducted in the WGTA, but, for this task, the opaque screen separating the monkey's compartment from the testing area had been removed. As is shown in Figure 3, the animal was faced with a test-board on which there was a box covering the foodwell. When pushed, this box tilted back, away from the animal, uncovering the foodwell. The reward (a peanut) was delivered to the foodwell through a tube attached to this box. The box remained open as long as the animal kept pushing it, but, because it was fixed to the test-board by means of a spring-hinge, it automatically returned to its original closed position when the animal stopped pushing it. Behind the test-board (at a distance of $26 \mathrm{~cm}$ ) and immediately below the one-way vision screen hiding the experimenter from view, there was a triangular box with an opening at the front through which the stimulus could be presented. Inside this apparatus, there was a revolving platform on which the stimulus to be shown was placed. Turning this platform resulted in the presentation or withdrawal of the stimulus.

The monkeys were initially trained to push back the manipulandum to receive peanuts. When they had mastered this task, they were tested for $2 \mathrm{~d}$ as follows: A stimulus (a matchbox) was presented $10 \times / \mathrm{d}$, and immediately following presentation of the stimulus, a peanut was delivered down the tube. After the animal had retrieved the peanut, the stimulus was withdrawn from the animal's view and the intertrial in-

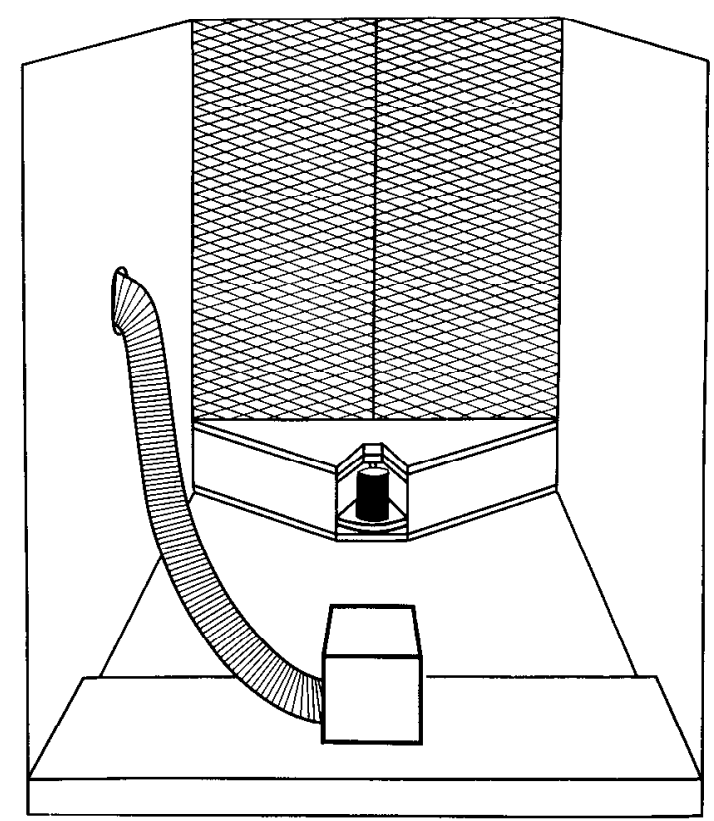

Figure 3. The monkey's view of the experimental arrangement. 


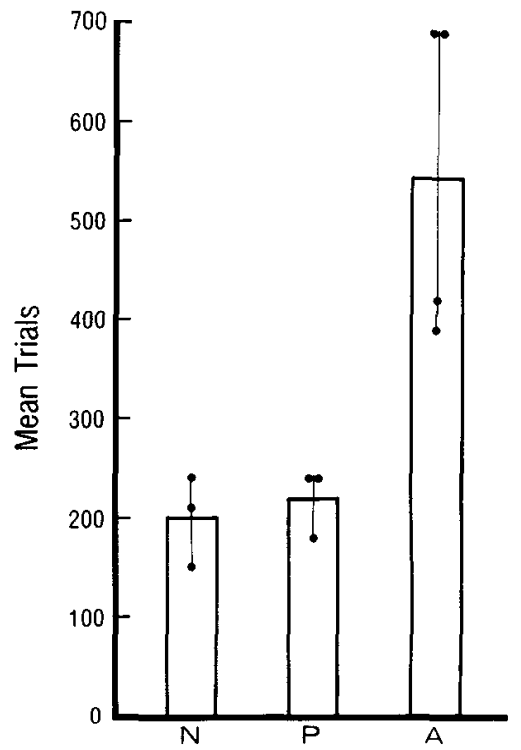

Figure 4. Performance on the visual, symmetrically reinforced, go, nogo task I by normal monkeys $(N)$, monkeys with periprincipalis lesions $(P)$, and monkeys with periarcuate lesions $(A)$. Solid circles, Individual scores for all the monkeys.

terval $(20 \mathrm{sec})$ was initiated. If the monkey responded (i.e., pushed open the box) during this period, the intertrial interval was prolonged for another $20 \mathrm{sec}$ and this process was continued until the monkey had failed to respond for $20 \mathrm{sec}$, when the next stimulus was shown. This procedure was designed to eliminate responses during the intertrial interval and to help the animals realize that there was a relationship between stimulus presentation and responding. It was also used in the other go, no-go tasks reported in this communication.

On the third day of training, the same stimulus was presented for 10 trials, but the reward was now delivered after $3 \mathrm{sec}$ unless the monkey responded earlier, in which case the reward was delivered immediately after the animal's response. Training continued in this fashion until the monkey had responded, before the $3 \mathrm{sec}$ had elapsed, to all 10 stimulus presentations on each of 2 consecutive d. As soon as this criterion was achieved, training was continued as follows: Ten trials were again administered per day with the same intertrial interval of $20 \mathrm{sec}$. On 5 of these trials, the stimulus was presented. If the monkey responded within $5 \mathrm{sec}$ of stimulus presentation, the reward was delivered. If he failed to respond during this period, the revolving platform was swung back, withdrawing the stimulus from the monkey's view and terminating the trial. This trial was then repeated until the monkey responded correctly (i.e., before the $5 \mathrm{sec}$ period had elapsed). On the other 5 trials (blank trials), the revolving stage was turned to open the box but no stimulus was presented. The stimulus-presentation apparatus remained open for $5 \mathrm{sec}$. If the monkey responded during these $5 \mathrm{sec}$, the trial was repeated and this was continued until the monkey stopped responding. The purpose of this part of the training was to teach the animals to look at what was presented and not to respond merely to the opening of the stimuluspresentation box. The order of presentation of the "blank" and "nonblank" trials was determined according to a random but balanced order (Gellermann schedule). Testing continued until the monkeys made one error or less on each of 3 consecutive $d$.

Following this preliminary training, testing on the go, no-go, symmetrically reinforced task started. In this task, the 2 stimuli used in the visual discrimination and reversal tests werc again utilized. Thirty trials ( 15 go and 15 no-go trials) were administered daily according to a random but balanced order (Gellermann schedule) and with the noncorrection technique. On the go trials, the red cross was presented and, if the monkey responded by opening the box within $5 \mathrm{sec}$, a peanut was delivered down the tube and the stimulus was removed from the subject's view. Failure to respond during this $5 \mathrm{sec}$ period led to termination of this trial and its scoring as incorrect. On the no-go trials, the yellow, oval-shaped object was presented for $5 \mathrm{sec}$. If the monkey withheld responding during this period, a peanut was delivered and the stimulus was withdrawn. If the monkey responded before the end of the $5 \mathrm{sec}$ period, the trial was terminated, no reward was delivered, and the trial was scored as incorrect. The intertrial interval was $20 \mathrm{sec}$. If the animal responded during this time, the stopwatch was reset and another interval of $20 \mathrm{sec}$ duration was initiated. This process was continued until the monkey had failed to respond for $20 \mathrm{sec}$, and the next trial was then administered. The experiment was terminated when the animals reached criterion, which was defined as 3 or fewer incorrect trials on each of 3 consecutive days of testing, or when 690 trials had been completed ( 23 d of testing).

\section{Results}

There were no significant differences between the groups on any aspect of the preliminary training needed to teach the animals to respond in the presence of a stimulus (a matchbox) and not merely to the opening of the box by means of which the stimuli were presented. There were, however, significant differences between the groups on the number of trials (see Fig. 4) required to learn to respond to the go stimulus and to withhold responding for $5 \mathrm{sec}$ to the no-go stimulus (Kruskal-Wallis test; $H=6.71, d f=2, p<0.013$ ). The periarcuate group required more trials to criterion than either the normal control (Mann-Whitney test; $\left.U=0, n_{1}=4, n_{2}=3, p=0.028\right)$ or the periprincipalis group $(U=0$, $n_{1}=4, n_{2}=3, p=0.028$ ). Two of the 4 animals that had periarcuate lesions failed to reach criterion within the limits of testing.

\section{Comment}

These results confirmed the prediction that monkeys with periarcuate lesions would be impaired in mastering a symmetrically reinforced go, no-go task. The normal performance of these animals on the retention of a visual discrimination task and the learning of its reversal (Experiment 1), in which the stimuli used were the same as those of the present experiment, suggests that the impairment on the go, no-go task cannot be attributed to visual discriminative difficulties with the stimuli or to perseverative deficits. The poor performance on the go, no-go task could, however, be interpreted as the result of a difficulty in learning to produce the correct response (go or no-go) to the appropriate stimulus.

\section{Experiment 3: visual discrimination $I I$ and reversal $I I$}

In this experiment, the animals were trained on a visual discrimination and its reversal with 2 new objects that were to become the stimuli in the asymmetrically reinforced go, no-go task (Experiment 4 ). The primary purpose of this experiment was to achieve comparability between the 2 different go, no-go tasks by using as stimuli, in both cases, objects that the animals had previously experienced in a standard visual discrimination task.

\section{Procedure}

The animals were tested for postoperative acquisition of a visual object discrimination and its reversal. The apparatus and testing procedure were the same as those used in Experiment 1. For the present visual discrimination, the positive stimulus was an orange-colored, wooden eggcup and the negative stimulus was a beige toothpick holder. For the reversal test, the toothpick holder became the positive stimulus and the eggcup the negative one.

\section{Results and comment}

As is shown in Table 1, there were no differences between the groups on either the initial learning (Kruskal-Wallis test; $H=1.02, d f-2$, n.s.) or the reversal of this visual discrimination $(H=4.62, d f=2$, n.s.). These findings are in agreement with the results of Experiment 1 and extend them by showing that not only can animals with periarcuate lesions retain a preoperatively learned visual discrimination, but they can also exhibit normal postoperative learning on such a task.

\section{Experiment 4: asymmetrically reinforced visual go, no-go task I}

\section{Procedure}

The apparatus and testing procedure were identical to those used in the symmetrically reinforced go, no-go task (Experiment 2), but with one critical difference: The animal was not rewarded for not responding on the "no-go" trials. The stimuli used in this experiment were the same as those used in Experiment 3. The orange-colored, wooden eggcup was 


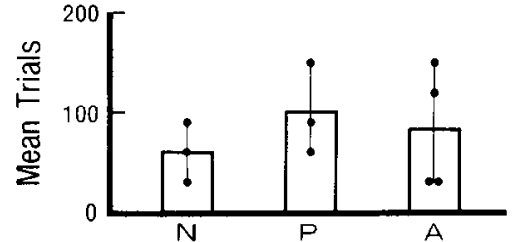

Figure 5. Performance on the visual, asymmetrically reinforced, go, no-go task I by normal monkeys $(N)$, monkeys with periprincipalis lesions $(P)$, and monkeys with periarcuate lesions $(A)$. Solid circles, Individual scores for all the monkeys.

the stimulus for the "go" response and the beige, toothpick holder for the "no-go" response.

\section{Results and comment}

The number of trials required by the various groups to reach criterion on this task are shown in Figure 5. There were no significant differences between the groups (Kruskal-Wallis test; $H=1.03, d f=2$, n.s.). The findings clearly indicated that, in marked contrast to the symmetrically reinforced go, no-go task, and as predicted, monkeys with periarcuatc lesions were not impaired on the asymmetrically reinforced go, no-go task. However, the asymmetrically reinforced task proved to be much easier than the symmetrically reinforced one. This, of course, raised the question of whether the same result would be obtained on a more difficult asymmetrical task. This question was addressed in the next experiment.

\section{Experiment 5: asymmetrically reinforced visual go, no-go task II}

In this experiment, the monkeys were trained on an asymmetrically reinforced go, no-go task that was intended to be more difficult than the task in Experiment 4. The animals were tested on this new task about 2 years postoperatively. In the period that intervened between Experiment 4 and the present experiment, the monkeys were tested on a motor conditional associative task and its control (see Petrides, 1982), as well as on the auditory go, no-go tasks described below in Experiments 7 and 8 .

\section{Procedure}

The stimuli were 10 objects that the monkeys had never seen before. Five of these objects were designated as the positive and the other 5 as the negative stimuli. The monkeys were rewarded for responding when one of the positive but not one of the negative stimuli was shown. Each one of these stimuli was presented 3 times during the daily testing session of 30 triais. All 10 objects were presented during the first, second, and third block of 10 trials. The order of presentation of the go and no-go trials was determined, as in the other experiments, according to a random but balanced order ( 5 go and 5 no-go trials occurred in a block of 10 trials). The positive and the negative objects were kept in 2 separate boxes. On any given trial, the experimenter drew randomly one of these stimuli from the appropriate box. At the end of a block of 10 trials, when all objects had been used, they were replaced in the boxes, and the next block of 10 trials was administered in the same way. Apart

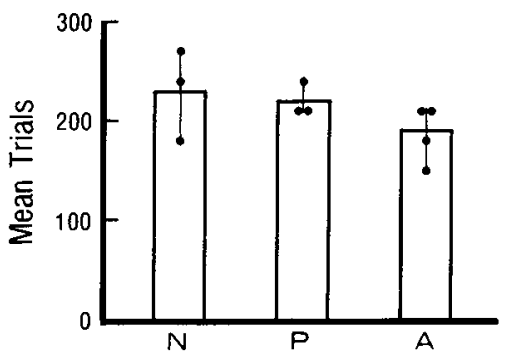

Figure 6. Performance on the visual, asymmetrically reinforced, go, no-go task II by normal monkeys $(N)$, monkeys with periprincipalis lesions $(P)$, and monkeys with periarcuate lesions $(A)$. Solid circles, Individual scores for all the monkeys.

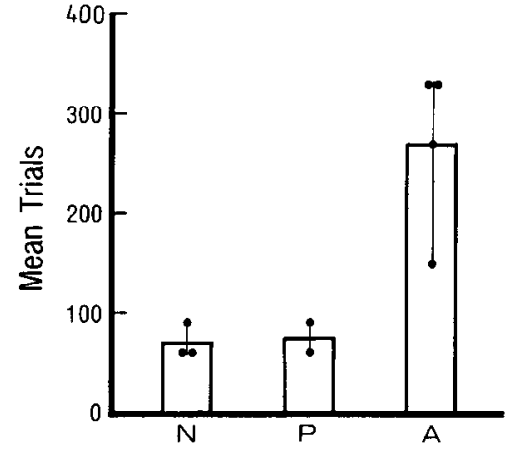

Figure 7. Performance on the visual, symmetrically reinforced, go, nogo task II by normal monkeys $(N)$, monkeys with periprincipalis lesions $(P)$, and monkeys with periarcuate lesions $(A)$. Solid circles, Individual scores for all the monkeys.

from these changes, the apparatus and testing procedure were identical to those used in Expcriment 4.

\section{Results and comment}

The number of trials required by the various animals to reach criterion on this task are shown in Figure 6. There were no significant differences between the groups (Kruskal-Wallis test; $H=2.60, d f=2, n . s$.) on this new, asymmetrically reinforced, go, no-go task, even though it proved to be much more difficult than the earlier asymmetrical task (Experiment 4) and at about the same level of difficulty as the symmetrically reinforced go, no-go task (Experiment 2). The present results, in conjunction with those of Experiment 4, show that asymmetrically reinforced visual go, no-go lasks can be learned normally by monkeys with periarcuate lesions.

\section{Experiment 6: symmetrically reinforced visual go, no-go task II}

Upon completion of Experiment 5, the monkeys were trained on a new symmetrically reinforced task, in order to determine whether the deficit seen after periarcuate lesions on such a task (Experiment 2) could be replicated about 2 years after operation and after considerable experience with go, no-go tasks.

One animal ( $\mathrm{P}-\mathrm{Wo}$.) from the periprincipalis group died from a large intrathoracic tumor, interfering with cardiac and respiratory functions, before testing on this experiment was initiated.

\section{Procedure}

The apparatus and testing procedure were identical to those used in the first symmetrically reinforced task (Experiment 2 ). There was no "preliminary training," however, in the present experiment because the animals were highly experienced with the testing situation and, therefore, such training was not necessary. The stimulus for the go response was a red toy truck and, for the no-go response, a blue battery.

\section{Results and comment}

This task proved to be a very easy one for all groups (see Fig. 7), clearly because the animals were very expcricnced with go, no-go tasks. Nevertheless, despite the simplicity of the task and the fact that the testing took place a long time after operation, the periarcuate animals showed a clear impairment in comparison with the control groups $\left(U=0, n_{1}=\right.$ 4, $n_{2}=3, p=0.028$ for periarcuate versus normal control; $U=0, n_{1}=$ $4, n_{2}=5, p=0.008$ for periarcuate versus normal control and periprincipalis groups).

\section{Experiment 7: symmetrically reinforced auditory go, no-go task}

Experiments 2 and 6 demonstrated that monkeys with periarcuate lesions were impaired in mastering visually cued, symmetrically reinforced go, no-go tasks. The present experiment investigated the question of whether a similar impairment could be demonstrated on such a task if the cues were auditory rather than visual. The fact that direct input reaches the periarcuate cortex not only from the visual but also from 


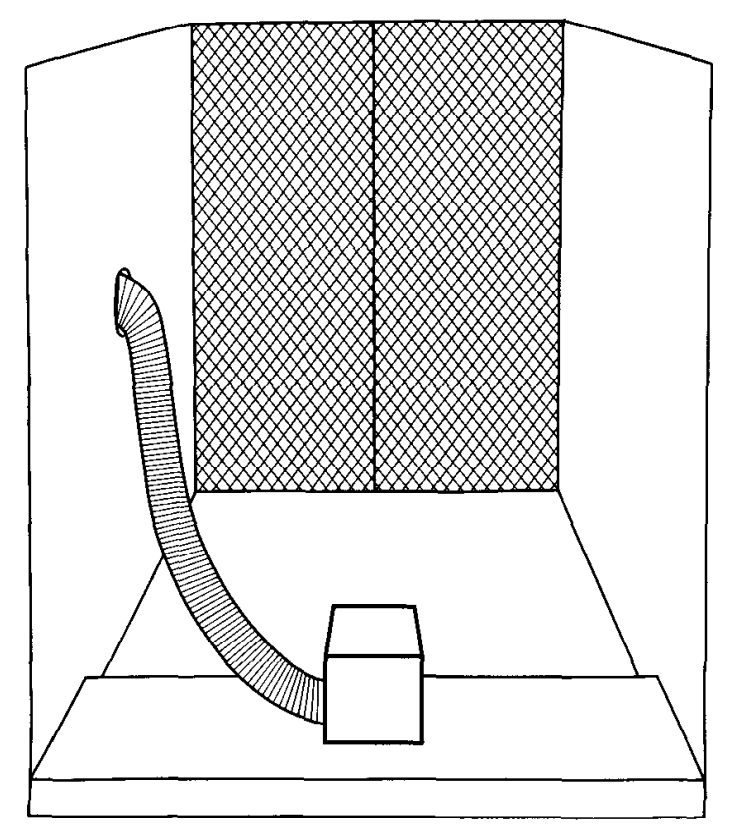

Figure 8. The monkey's view of the experimental arrangement for the auditory go, no-go task.

the auditory and the somaesthetic system suggests that the role of this part of the frontal cortex in conditional learning may not be modalityspecific (Petrides, 1982).

Deficits on an auditory, symmetrically reinforced, go, no-go task have been reported in the monkey after large lesions of the dorsolateral frontal cortex that included the sulcus principalis, the inferior convexity, and parts of the periarcuate cortex (Gross, 1963; Symmes, 1967; Weiskrantz and Mishkin, 1958). The role of the periarcuate cortex in such a task, however, cannot be established from the above studies since the lesions were not restricted to it, and, therefore, the deficits observed could have been due to the perseverative interference that is known to result from large frontal lesions that include the inferior part of the dorsolateral convexity. An investigation by Gross and Weiskrantz (1962) suggested that the deficit on this auditory task observed after large frontal lesions may be dissociable from the delayed-response deficit that occurs after lesions restricted to the region of the sulcus principalis.

\section{Procedure}

In this experiment, the monkey was faced with the same response manipulandum that was used in the visual go, no-go tasks except that the apparatus used to present the visual stimulus was removed from the animal's view (see Fig. 8). The auditory stimuli were delivered through a single speaker placed above the monkey's testing cage on the roof of the WGTA.

Before testing on the go, no-go task was initiated, the following preliminary training was administered to draw the attention of the animals to the fact that there was a relationship between the presence of a tone and the production of a response. During this preliminary training, 20 trials were given/d. A trial was initiated when a tone $(1200 \mathrm{~Hz}, 74 \mathrm{~dB})$ was presented through the speaker above the monkey's testing cage. If the monkey responded within $5 \mathrm{sec}$ by pushing back the manipulandum, the reward was delivered down the tube and the tone was turned off. If the monkey failed to respond within $5 \mathrm{sec}$, the tone was turned off and the trial was repeated after an intertrial interval of $10 \mathrm{sec}$. Every time the monkey responded during this interval, the interval was prolonged for an additional $10 \mathrm{sec}$ period. This procedure, which was also used throughout testing on this task, and the other go, no-go tasks, was designed to eliminate intertrial responding and to emphasize the relation between responses and the presence of the stimulus. The preliminary training was terminated when the animal had responded correctly on each of 20 consecutive trials. This training was completed in $2-4 \mathrm{~d}$ by all animals, there being no significant differences among the 3 groups.

In the go, no-go training, which was instituted immediately after completion of the preliminary testing, one of 2 tones was presented on each trial. The intensity of these 2 tones, as measured in the monkey's

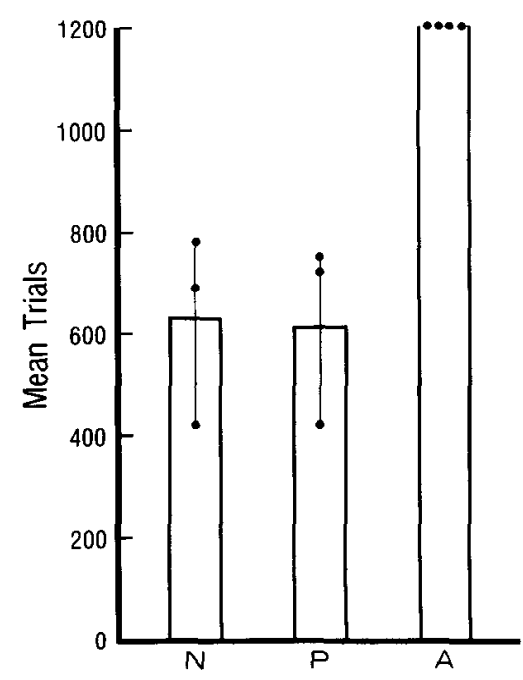

Figure 9. Performance on the auditory, symmetrically reinforced, go, no-go task by normal monkeys $(N)$, monkeys with periprincipalis lesions $(P)$, and monkeys with periarcuate lesions $(A)$. Solid circles, Individual scores for all the monkeys.

compartment, was set at $74 \mathrm{~dB}$. On the go trials, a $400 \mathrm{~Hz}$ tone was presented and, if the animal responded within $5 \mathrm{sec}$ of stimulus presentation by pushing open the manipulandum, a peanut was delivered down the lube. If the animal did not respond within this $5 \mathrm{sec}$ period, the tone was terminated and the next trial administered. On the go, nogo trials, an $800 \mathrm{~Hz}$ tone was presented and, if the animal refrained from responding for $5 \mathrm{sec}$, he was rewarded by the delivery of the peanut down the tube. If the animal responded during the $5 \mathrm{sec}$ that the 800 $\mathrm{Hz}$ tone was on, the tone was turned off and no reward was delivered. Thirty trials ( 15 go and 15 no-go) were administered per day according to a random but balanced sequence (Gellermann schedule). A noncorrection procedure was followed throughout training, i.e., irrespective of whether an animal had responded correctly or not on any given trial, the next trial, go or no-go, was administered. Testing was continued to a criterion of 27 or more out of 30 correct responses on each of 3 consecutive $\mathrm{d}$ or to a maximum of 1200 trials.

\section{Results}

No significant differences between the groups on any aspect of the preliminary training were observed. There were, however, significant differences between groups on the number of trials needed to reach criterion in the main part of the experiment in which the animals had to learn to respond to a $400 \mathrm{~Hz}$ tone and to withhold responding to an $800 \mathrm{~Hz}$ tone (Kruskal-Wallis test; $H=6.55, d f=2, p<0.05$ ). As can be seen in Figure 9, none of the 4 animals with periarcuate lesions was able to reach criterion within the limits of testing, in contrast to the 3 animals with periprincipalis lesions, which performed as well as the normal control monkeys. The periarcuate group was significantly impaired in comparison with the normal control group (Mann-Whitney test; $U=$ $0, n_{1}=4, n_{2}=3, p=0.028$ ), while the periprincipalis group did not differ from the control group ( $U=4.5, n_{1}=3, n_{2}=3$, n.s.).

\section{Comment}

These data demonstrate that animals with periarcuate lesions are impaired not only on visually cued, symmetrically reinforced go, no-go tasks, but also on comparable tasks in which the cues are auditory. The auditory stimuli used in the present experiment were the same as those used in a different auditory go, no-go task (Van Hoesen et al., 1980), in which monkeys with periarcuate lesions, larger than those studied here, were found to perform as well as normal control animals. In the auditory go, no-go task used by Van Hoesen et al. (1980), the animals were rewarded when they responded to the tone that was designated as "positive," but were not rewarded when they responded to the "negative" tone. That task was therefore comparable to the asymmetrically reinforced go, no-go tasks administered in this series of experiments, and the fact that animals with periarcuate lesions performed normally on it is consistent with the argument developed in the present com- 


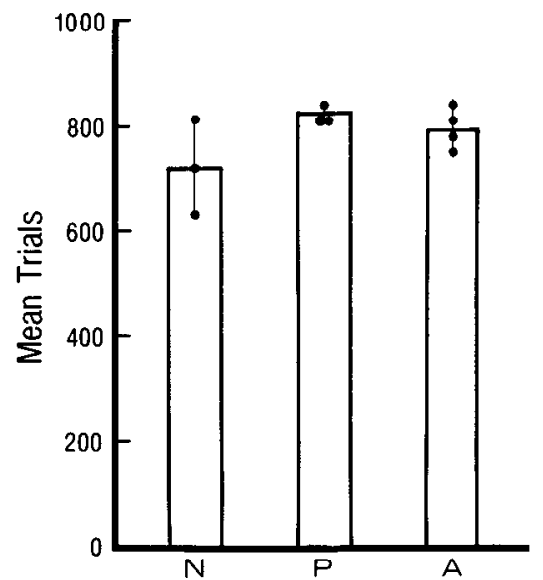

Figure 10. Performance on the auditory, asymmetrically reinforced, go, no-go task by normal monkeys $(N)$, monkeys with periprincipalis lesions $(P)$, and monkeys with periarcuate lesions $(A)$. Solid circles, Individual scores for all the monkeys.

munication. In addition, the normal performance of monkeys with periarcuate lesions on the auditory task used by Van Hoesen et al. (1980) argues against any attempt to reduce the deficit observed in the present experiment in terms of difficulty in discriminating between the auditory stimuli.

\section{Experiment 8: asymmetrically reinforced auditory go, no-go task}

As pointed out above, Van Hoesen et al. (1980) found that monkeys with periarcuate lesions were able to learn, at a normal rate, an auditory task that was an asymmetrically reinforced go, no-go task. Nevertheless, it seemed important to investigate the performance on such a task of the present group of animals with periarcuate lesions, which had demonstrated severe impairments on the symmetrically reinforced auditory task (Experiment 7).

\section{Procedure}

Before testing on the go, no-go task was initiated, the monkeys received preliminary training with a new tone $(200 \mathrm{~Hz}, 74 \mathrm{~dB})$. In this training, 20 trials/d were administered, during which the monkeys had to push open the manipulandum when the tone was presented. When this preliminary training, which was completed within $2 \mathrm{~d}$ by all animals, had been finished, the monkeys were tested on 15 go and 15 no-go trials/d, presented according to a random but balanced sequence. On the go trials, the "positive" tone $(1000 \mathrm{~Hz}, 74 \mathrm{~dB})$ was presented and the animals were rewarded if they responded to it. On the no-go trials, the "negative" tone $(600 \mathrm{~Hz}, 74 \mathrm{~dB})$ was presented and the animals were not rewarded if they responded to it. The apparatus and testing procedure for both the preliminary training and the main part of the experiment were identical to those used in the symmetrically reinforced go, no-go task (Experiment 7), with one critical difference: In the symmetrically reinforced task, the animals were rewarded if they correctly withheld responding on the no-go trials for $5 \mathrm{sec}$.

\section{Results and comment}

The trials required by the various groups of animals to learn this task are illustrated in Figure 10. There were no significant differences between the groups on this task (Kruskal-Wallis test; $H=3.12, d f=2$, n.s.). The present data, in agreement with previous findings (Van Hoesen et al., 1980), demonstrate that damage to the periarcuate cortex does not impair the acquisition of an acoustically cued, asymmetrically reinforced go, no-go task, even though it does give rise to severe impairments when both the go and the no-go responses are rewarded when correctly produced, as was demonstrated in Experiment 7 . The findings on the auditory go, no-go tasks are consistent with those obtained on the visual go, no-go tasks studied in the present series of experiments.

\section{Discussion}

Monkeys with periarcuate lesions were impaired in mastering symmetrically reinforced, but not asymmetrically reinforced, go, no-go tasks. In the asymmetrically reinforced tasks, the animal had to learn to respond in the presence of the positive stimulus because such responses were rewarded, whereas responses in the presence of the negative stimulus were extinguished through non-reward. In the asymmetrical go, no-go tasks used in the present series of experiments, the key requirement was to learn, on the basis of differential reinforcement, when to emit the response. In the symmetrically reinforced tasks, on the other hand, learning could not have been based on differential reinforcement since both the production and the withholding of the responses were rewarded when they occurred in the presence of the appropriate stimuli. Performance had to be based, instead, on a conditional rule specifying the appropriate response to each one of a set of exteroceptive stimuli. The power of the present demonstration that animals with periarcuate lesions failed only the symmetrically reinforced tasks derives from the fact that the 2 types of tasks investigated were identical to each other, the only difference being in the testing procedures involved. Thus, the normal performance of the monkeys with periarcuate lesions on the asymmetrical tasks rules out interpretations of the deficit observed on the symmetrical tasks as due to the method of stimulus presentation, the type of response required, difficulties in discriminating between the stimuli, or impairments in arousal, attention, eye movements, or motivation. Similarly, the normal performance of these monkeys on the visual discrimination tasks and their reversals provides further evidence that the impairments observed in the symmetrical tasks could not have been due to difficulties in visual discrimination or perseverative interference, the latter being characteristic of monkeys with lesions to the inferior convexity of the frontal lobe (e.g., Iversen and Mishkin, 1970). Finally, it should be pointed out that there is some evidence indicating that performance on the type of asymmetrical tasks used in the present investigation is impaired after damage to the medial frontal cortex in the dog (Dabrowska, 1971) and the orbitofrontal cortex in the monkey (Lawicka et al., 1975).

The deficit on the symmetrically reinforced tasks by monkeys with periarcuate lesions supports the hypothesis that this region of the frontal cortex may be critically involved in the learning and performance of conditional tasks, in which the animal must learn to select the correct response to each one of a set of exteroceptive stimuli (Petrides, 1982). It does not, however, support attempts to reduce the impairment of such monkeys on conditional position-response tasks to spatial difficulties. The present findings are also in agreement with the demonstration that damage to the periarcuate cortex impairs learning on a conditional task requiring selection between two nonspatial responses (Petrides, 1985b) and on a motor conditional task (Petrides, 1982). Postoperative retention on a motor conditional task has also been shown to be impaired after lesions restricted to area 6 (Halsband and Passingham, 1982). In all these tasks, the animals had to produce one or another response (e.g., make movement $\mathrm{X}$ or $\mathrm{Y}$, select nonspatial stimulus $\mathrm{X}$ or $\mathrm{Y}$, etc.), conditional upon the presentation of signals $A$ or $B$ that were physically separate from the responses. It is important to emphasize this point because a number of situations may appear superficially to require conditional learning but, in fact, do not. These are often situations where the response manipulandum is also the stimulus. There is a large body of evidence in the animal learning literature showing that, in such tasks, the animals tend to learn to approach, avoid, or manipulate in particular ways individual stimuli, rather than learn to select between responses $\mathrm{X}$ and $\mathrm{Y}$, conditional upon presentation of arbitrary signals (see Mackintosh, 1974). In these latter situations, one would expect monkeys with damage to the periarcuate cortex to perform normally. There is some evidence in support of this point in work with selective frontal lesions in monkeys (Gross and Weiskrantz, 1962; Passingham, 1985; Petrides, in press; 
Stamm, 1973) and with complete frontal lobectomies in dogs (Lawicka and Konorski, 1959).

As was discussed above, the important difference between the asymmetrically and symmetrically reinforced go, no-go tasks studied in the present series of experiments is that responses in the presence of the negative stimuli in the asymmetrical, but not the symmetrical, tasks can be extinguished through lack of reinforcement. The important issue here is whether a task can be learned on the basis of strengthening some responses through reward and eliminating others through non-reward, or whether the learning demands of the task are such that the animal must acquire and perform tasks according to a conditional rule. This point must be emphasized because there are tasks in which, even though reinforcement is asymmetrical, the animal can only achieve strict criterion by learning a conditional rule. One such task is the visual-auditory compound task whose acquisition (Petrides and Iversen, 1978) and retention (Van Hoesen et al., 1980) has been shown to be impaired after periarcuate lesions. In this task, the monkeys were rewarded for responding when a visual and an auditory stimulus were presented together, but not when these stimuli were presented alone. In this situation, the withholding of responses, when the stimuli were presented separately, could not simply have been the result of elimination of responses through non-reward, since responding to these same stimuli was rewarded when they were presented together. Thus, the mechanism of extinction of non-rewarded responses, which is intact after periarcuate lesions, as has been demonstrated in the present experiments, could not lead to criterion performance on the task. The animals, instead, had to learn and perform according to the conditional rule: If the 2 stimuli occur together, respond; if either one is presented, withhold responding. Similarly, the go, no-go alternation task used in the investigation by Goldman et al. (1971) is not related, in terms of learning requirements, to the asymmetrically reinforced go, no-go tasks used in the present series of experiments-criterion performance on the alternation task, unlike that of the present asymmetrical go, no-go tasks, cannot be the result of learning to emit the response in the presence of an exteroceptive stimulus (the "positive" cue) that leads to reward, and not in the presence of another exteroceptive stimulus (the "negative" cue) that does not.

Recent single-cell recording investigations have shown, in agreement with predictions from the available anatomical work, that cells in the periarcuate region of the frontal lobe respond to visual (Gentilucci et al., 1983; Godschalk et al., 1981; Kubota and Hamada, 1978; Mohler et al., 1973; Rizzolatti et al., 198 la) and somaesthetic (Rizzolatti et al., 1981b) stimuli and, furthermore, that many cells in this region exhibit modulations of their response patterns in relation to the "instructional" significance of stimuli rather than to their physical characteristics (Weinrich and Wise, 1982; Weinrich et al., 1984; Wise and Mauritz, 1985). This work is thus consistent with the hypothesis, in favor of which support was provided in the present communication, that the periarcuate cortex is critically involved in conditional learning situations where, out of a pool of responses, the appropriate one has to be selected on the basis of a given stimulus.

\section{References}

Astruc, J. (1971) Corticofugal connections of area 8 (frontal eye field) in Macaca mulatta. Brain Res. 33: 241-256.

Barbas, H., and M.-M. Mesulam (1981) Organization of afferent input to subdivisions of area 8 in the rhesus monkey. J. Comp. Neurol. 200: 407-431.

Chavis, D. A., and D. N. Pandya (1976) Further observations on corticofrontal connections in the rhesus monkey. Brain Res. 117:369386.
Dabrowska, J. (1971) Dissociation of impairment after lateral and medial prefrontal lesions in dogs. Science 171: 1037-1038.

Dabrowska, J. (1972) On the mechanism of go-no go symmctrically reinforced task in dogs. Acta Neurobiol. Exp. 32: 345-359.

Gentilucci, M., C. Scandolara, I. N. Pigarev, and G. Rizzolatti (1983) Visual responses in the postarcuate cortex (area 6) of the monkey that are independent of eye position. Exp. Brain Res. 50: 464-468.

Godschalk, M., R. N. Lemon, H. G. T. Nijs, and H. G. J. M. Kuypers (1981) Behaviour of neurons in monkey peri-arcuate cortex and precentral cortex before and during visually guided arm and hand movements. Exp. Brain Res. 44: 113-116.

Godschalk, M., R. N. Lemon, H. G. J. M. Kuypers, and H. K. Ronday (1984) Cortical afferents and efferents of monkey postarcuate area: An anatomical and electrophysiological study. Exp. Brain Res. 56: 410-424.

Goldman, P. S., and H. E. Rosvold (1970) Localization of function within the dorsolateral prefrontal cortex of the rhesus monkey. Exp. Neurol. 27: 291-304.

Goldman, P. S., H. E. Rosvold, B. Vest, and T. W. Galkin (1971) Analysis of the delayed-alternation deficit produced by dorsolateral prefrontal lesions in the rhesus monkey. J. Comp. Physiol. Psychol. 77: 212-220.

Gross, C. G. (1963) A comparison of the effects of partial and total lateral frontal lesions on test performance by monkeys. J. Comp. Physiol. Psychol. 56: 41-47.

Gross, C. G., and L. Weiskrantz (1962) Evidence for dissociation of impairment on auditory discrimination and delayed response following lateral frontal lesions in monkeys. Exp. Neurol. 5: 453-476.

Halsband, U., and R. Passingham (1982) The role of premotor and parietal cortex in the direction of action. Brain Res. 240: 368-372.

Iversen, S. D., and M. Mishkin (1970) Perseverative interference in monkeys following selective lesions of the inferior prefrontal convexity. Exp. Brain Res. 11: 376-386.

Konorski, J. (1972) Some hypotheses concerning the functional organization of prefrontal cortex. Acta Neurobiol. Exp. 32: 595-613.

Konorski, J. (1975) The role of prefrontal control in the programming of motor behavior. In Efferent Urganization and the Integration of Behavior, J. D. Maser, ed., pp. 175-201, Academic, New York.

Kubota, K., and I. Hamada (1978) Visual tracking and neuron activity in the post-arcuate area in monkeys. J. Physiol. (Paris) 74: 297-312.

Kuypers, H. G. J. M., and D. Lawrence (1967) Cortical projections to the red nucleus and the brain stem in the rhesus monkey. Brain Res. 4: 151-188.

Lawicka, W., and J. Konorski (1959) Physiological mechanism of delayed reactions. III. The effects of prefrontal ablations on delayed reactions in dogs. Acta Biol. Exp. 19: 221-231.

Lawicka, W., M. Mishkin, and H. E. Rosvold (1975) Dissociation of deficits on auditory tasks following partial prefrontal lesions in monkeys. Acta Neurobiol. Exp. 35: 581-607.

Mackintosh, N. J. (1974) The Psychology of Animal Learning, Academic, London.

Matsumura, M., and K. Kubota (1979) Cortical projection to handarm motor area from post-arcuate area in macaque monkeys: A histological study of retrograde transport of horseradish peroxidase. Neurosci. Lett. 11: 241-246.

Milner, A. D., N. P. Foreman, and M. A. Goodale (1978) Go-left goright discrimination performance and distractibility following lesions of prefrontal cortex or superior colliculus in stumptail macaque. Neuropsychologia 16: 381-390.

Milner, B. (1982) Some cognitive effects of frontal-lobe lesions in man. Phil. Trans. R. Soc. Lond. [Biol.] 298: 211-226.

Mohler, C. W., M. E. Goldberg, and R. H. Wurtz (1973) Visual receptive fields of frontal eye field neurons. Brain Res. 61: 385-389.

Muakkassa, K. F., and P. L. Strick (1979) Frontal lobe inputs to primate motor cortex: Evidence for four somatotopically organized "premotor" areas. Brain Res. 177: 176-182.

Pandya, D. N., and H. G. J. M. Kuypers (1969) Cortico-cortical connections in the rhesus monkey. Brain Res. 13: 13-36.

Pandya, D. N., and L. A. Vignolo (1971) Intra- and interhemispheric projections of the precentral, premotor, and arcuate areas in the rhesus monkey. Brain Res. 26: 217-233.

Pandya, D. N., P. Dye, and N. Butters (1971) Efferent cortico-cortical projections of the prefrontal cortex in the rhesus monkey. Brain Res. 31: $35-46$. 
Passingham, R. E. (1985) Cortical mechanisms and cues for action. Phil. Trans. R. Soc. Lond. [Biol.] 308: 101-111.

Petrides, M. (1982) Motor conditional associative-learning after selective prefrontal lesions in the monkey. Behav. Brain Res. 5: 407413.

Petrides, M. (1985a) Deficits on conditional associative-learning tasks after frontal- and temporal-lobe lesions in man. Neuropsychologia 23: $601-614$

Petrides, M. (1985b) Deficits in nonspatial conditional associative learning after periarcuate lesions in the monkey. Behav. Brain Res. 16: $95-101$.

Petrides, M. (in press) Conditional learning and the primate frontal cortex. In The Frontal Lobes Revisited, E. Perecman, ed., IRBN, New York.

Petrides, M., and S. D. Iversen (1978) The effect of selective anterior and posterior association cortex lesions in the monkey on performance of a visual-auditory compound discrimination test. Neuropsychologia 16: 527-537.

Petrides, M., and D. N. Pandya (1984) Projections to the frontal cortex from the posterior parietal region in the rhesus monkey. J. Comp. Neurol. 228: 105-116.

Podbros, I. Z., J. S. Stamm, and F. J. Denaro (1980) Associative function of the arcuate segment of the monkey's prefrontal cortex. Physiol. Behav. 24: 103-109.

Rizzolatti, G., C. Scandolara, M. Matelli, and M. Gentilucci (1981a) Afferent properties of periarcuate neurons in macaque monkeys. II. Visual responses. Behav. Brain Res. 2: 147-163.

Rizzolatti, G., C. Scandolara, M. Matelli, and M. Gentilucci (1981b)
Afferent properties of periarcuate neurons in macaque monkeys. I. Somatosensory responses. Behav. Brain Res. 2: 125-146.

Rosenkilde, C. E., H. E. Rosvold, and M. Mishkin (1981) Time discrimination with positional responses after selective prefrontal lesions in monkeys. Brain Res. 210: 129-144.

Stamm, J. S. (1973) Functional dissociation between the inferior and arcuate segments of dorsolateral prefrontal cortex in the monkey. Neuropsychologia 11: 181-190.

Symmes, D. (1967) Behavioral and electrophysiological studies on auditory functions of the frontal lobe in monkeys. J. Audit. Res. 7: 335-351.

Van Hoesen, G. W., D. N. Pandya, and N. Butters (1972) Cortical afferents to the entorhinal cortex of the rhesus monkey. Science 175: 1471-1473.

Van Hoesen, G. W., B. A. Vogt, D. N. Pandya, and T. M. McKenna (1980) Compound stimulus differentiation behavior in the rhesus monkey following periarcuate ablation. Brain Res. 186: 365-378.

Weinrich, M., and S. P. Wise (1982) The premotor cortex of the monkey. J. Neurosci. 2: 1329-1345.

Weinrich, M., S. P. Wise, and K.-H. Mauritz (1984) A neurophysiological study of the premotor cortex in the rhesus monkey. Brain 107 . $385-414$

Weiskrantz, L., and M. Mishkin (1958) Effects of temporal and frontal cortical lesions on auditory discrimination in monkeys. Brain 81 . $406-414$.

Wise, S. P., and K.-H. Mauritz (1985) Set-related neuronal activity in the premotor cortex of rhesus monkeys: Effects of changes in motor set. Proc. R. Soc. Lond. [Biol.] 223: 331-354. 